\title{
Rhinovirus infections
}

\author{
P. G. HigGins \\ Virus Reference Laboratory, Central Public Health Laboratory, \\ Colindale Avenue, London NW9 5 HT
}

\begin{abstract}
Summary
One hundred and seventy-five rhinoviruses were isolated from $4.4 \%$ of patients in the general practice survey and 44 from $1.8 \%$ of children in the hospital survey. Isolations were made at all times of the year and although isolated most frequently from cases of common cold rhinoviruses were also found in association with other respiratory syndromes.
\end{abstract}

The rhinoviruses were the most recently described agents to be sought in the MRC/PHLS studies. Not until 1960 was a practical routine method for their detection described (Tyrrell \& Parsons, 1960) with the result that, at the commencement of the studies in October 1964, few reports were available based on a substantial number of isolates. However, rhinoviruses had been shown to be associated with naturally occurring respiratory infections (e.g. Kendall, Bynoe \& Tyrrell, 1962) and there was evidence suggesting they might be the commonest agents to be isolated from such illnesses in the general population (Higgins, Boston \& Ellis, 1964).

During a collaborative survey of acute infections of the respiratory tract (MRC, 1965) which immediately preceded the present studies, seventyeight rhinoviruses were isolated and only enteroviruses (ninety-three) and adenoviruses (eighty-six) were encountered with greater frequency. The rhinoviruses were shown to be present at all seasons and, while commonly being isolated from patients with a common cold, they were also found in a small proportion of patients diagnosed as suffering from other forms of respiratory illness.

\section{Results of MRC/PHLS surveys}

Rhinoviruses were isolated from 175 of 3966 patients $(4.4 \%)$ sampled in the general practice study and only parainfluenza virus infections (190) were more commonly diagnosed. Forty-four strains were detected in specimens from $2418(1.8 \%)$ children admitted to hospital and proven rhinovirus infections were seen less often than infections with any other group of viruses in these patients.

Rhinoviruses were isolated from two of 280 children $(0.7 \%)$ without respiratory symptoms at the same time as eleven strains were recovered from 648 patients $(1 \cdot 7 \%)$ with respiratory illness-a difference that is not significant at the $5 \%$ level.

\section{Season}

Rhinoviruses were isolated at all seasons in both surveys (see Figs. $1 \& 2$, Poole \& Tobin, this symposium). Although there were fluctuations, from month to month, in the frequency with which these viruses were detected in individual practices the numbers isolated were too small to determine if this variation was significant.

\section{Age}

Rhinoviruses were isolated infrequently from children admitted to hospital under 1 year old $(1.6 \%)$ or those between 1 and 4 years of age $(1.6 \%$ but were associated with a greater proportion ( $3.5 \%$ ) of respiratory illness in older children (Fig. 1). In general practice (Fig. 2) the isolation rates were higher for children under 5 years $(4.1 \%)$ and adults $(5 \cdot 8 \%)$ than for school children $(2 \cdot 3 \%)$.

\section{Diagnosis}

Figs. 1 and 2 also show, by age group, the proportion of cases within each of the various diagnostic categories which was shown to be rhinovirus infections. In the general practice survey a rhinovirus was isolated from at least one in every forty cases sampled irrespective of the diagnosis but of children admitted to hospital only those with colds gave as high an isolation rate. However, of children 5-14 years old in hospital, between 4 and $6 \%$ with colds, croup, bronchitis and pneumonia were found to be excreting rhinoviruses.

In general practice higher isolation rates were found in patients aged 5-15 years with croup (8.3\%), and in older patients suffering from colds $(8.7 \%)$, laryngitis and croup $(6 \cdot 2 \%)$ and tracheitis $(8.3 \%)$. Rhinoviruses accounted for approximately half the viruses isolated from adult patients with colds and laryngitis and over a third of those with tracheitis and bronchitis. 


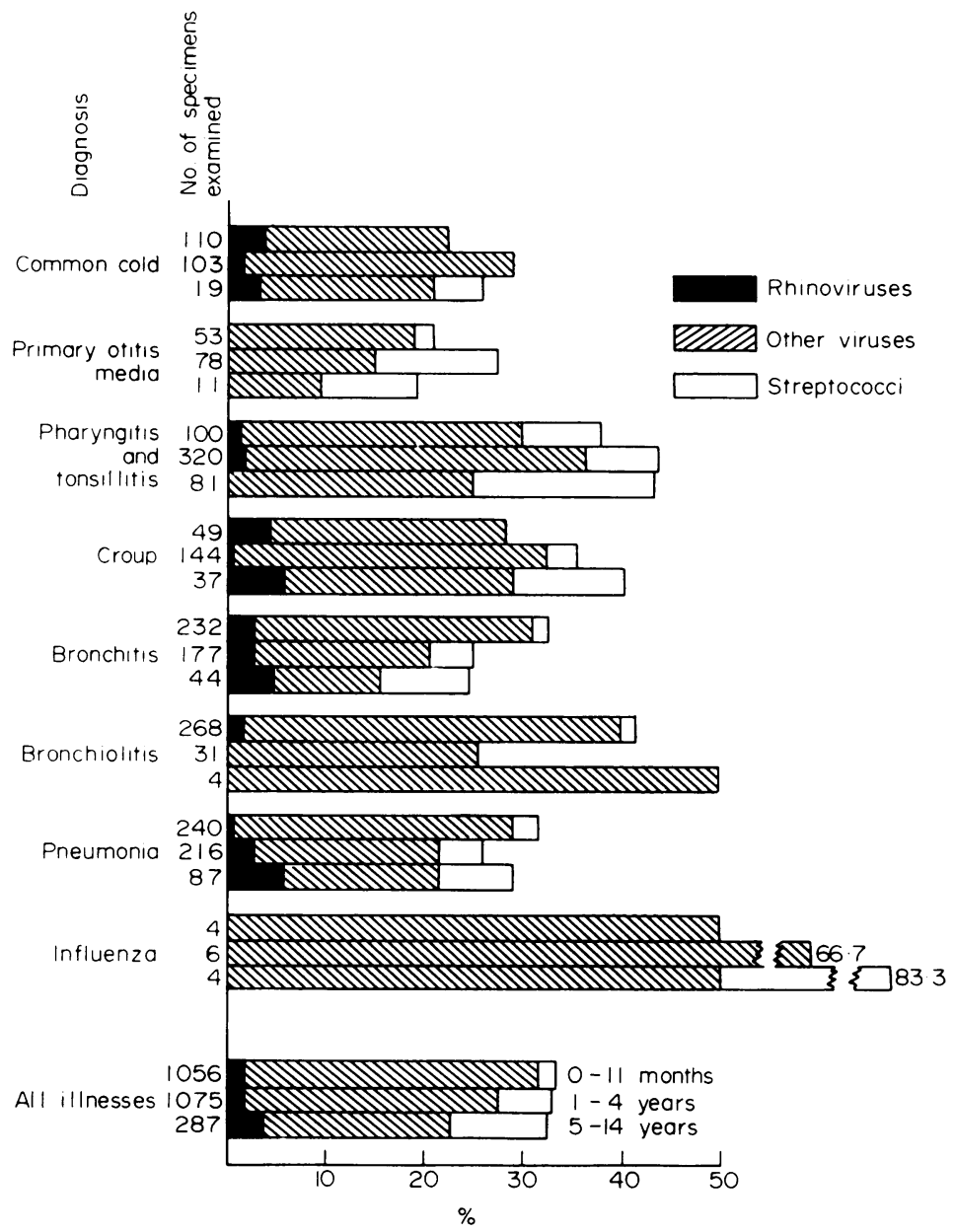

FIG. 1. The distribution of rhinoviruses by age and clinical illness. Hospital survey.

\section{Clinical features}

The four commonest clinical features of the 232 rhinovirus infections seen in the two surveys combined were: coryza, cough, red or sore throat and fever (Figs. 3 and 4). Headache, general aches and hoarseness were more common in adults (between 30 and $40 \%$ of cases) than in children and the converse was true for vomiting and dyspnoea.

\section{Laboratory findings}

Those tissue cultures purposely included in the survey to detect rhinoviruses did so in approximately $3 \%$ of the specimens examined, varying from $2.9 \%$ in human embryo kidney cells to $3.5 \%$ in cultures of HEL7. Rhinoviruses were isolated from twentynine $(0.4 \%)$ of the specimens examined in monkey kidney cells but other cultures were of no practical use for this purpose (see Poole \& Tobin, Table 3 this symposium).

The duration of the illness at the time of swabbing had no influence on the ability to isolate rhinoviruses from the specimens taken (see Poole \& Tobin, Table 4, this symposium).

\section{Discussion}

Rhinovirus infections were found to be common in the general population and to occur at all seasons of the year. The increased prevalence of these viruses during the autumn or early winter reported by other workers (Hamre, Connolly \& Procknow, 1966; Higgins, 1967) was not observed, probably because of the small number of strains isolated in any one laboratory. The lower isolation rate for rhinoviruses in the hospital study can be attributed to the mild nature 


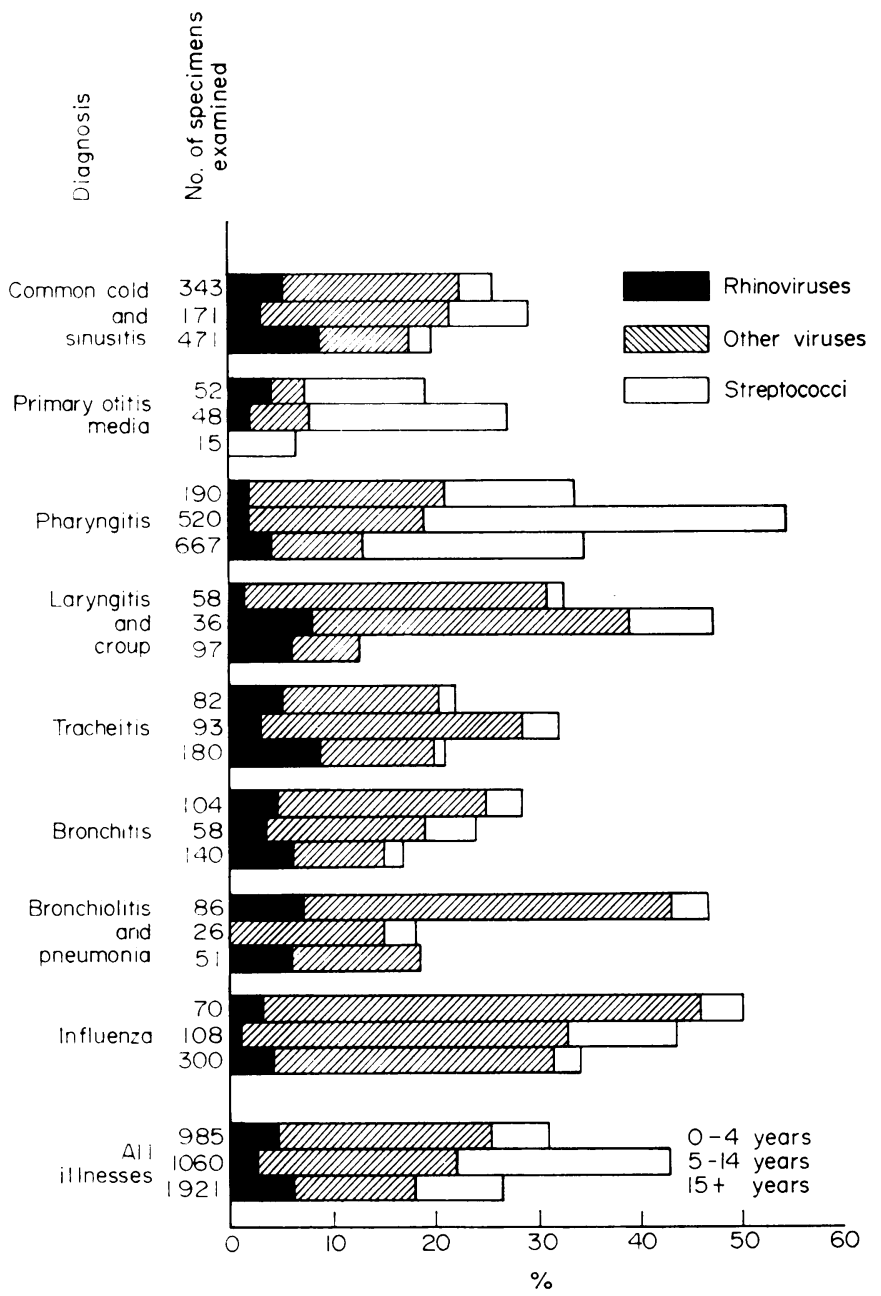

Fig. 2. The distribution of rhinoviruses by age and clinical illness. General practice survey.

of most infections while the increasing importance of these viruses with age, is most likely the result of a build up of immunity to a number of the other respiratory pathogens.

Although rhinoviruses were the commonest diagnosed cause of the common cold a significant proportion of all other respiratory tract infections was found to be associated with rhinoviruses, especially croup, laryngitis, bronchitis and tracheitis.

The four commonest clinical features of rhinovirus infection-coryza, cough, sore throat and feverare not diagnostic and also occur with greater frequency than the other features sought in a number of conditions, e.g. both influenza virus $A$ and adenovirus infections in children in hospital.
It was realized at the outset that, because of the difficulty in obtaining human embryonic kidneg few specimens would be examined in both this tissue and a sensitive strain of diploid fibroblasts This proved to be so and together with the failure to examine 730 specimens $(11.1 \%)$ in either cell. system could account, in part, for the relativeliso small number of isolations made (Higgins, 1966a?. More recently it has been shown that many rhindo viruses will only grow in tissue culture after passage in organ culture of human embryonic ciliate respiratory epithelium (Tyrrell \& Bynoe, 1966s Higgins, Ellis \& Woolley, 1969) and the number of isolations reported in this study must be an undef estimate of the rhinovirus infections sampled. Some 


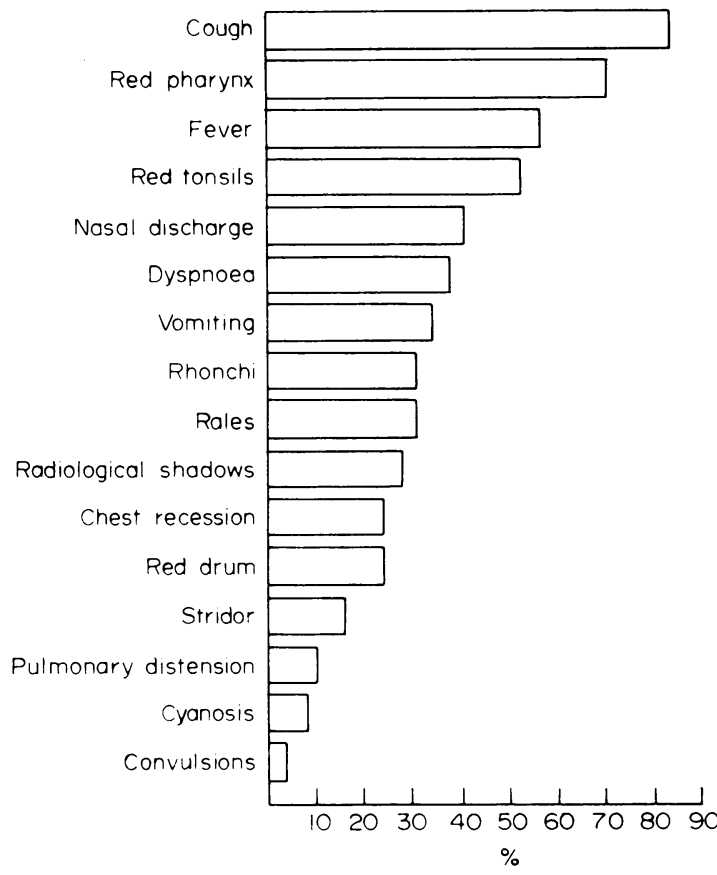

FIG. 3. Clinical features of forty-nine rhinovirus infections in children. Hospital survey.

idea of this deficiency can be judged by comparing the isolation rate in the general practice study $(4.4 \%)$ with the recently reported finding that $15 \%$ of similar specimens, which were negative on examination in tissue culture, yielded viruses after passage in organ cultures; $80 \%$ of these were rhinoviruses (Higgins \& Ellis, 1973).

It will be appreciated that it was not possible to justify the expenditure of labour and materials involved in any attempt to serotype those rhinoviruses which were isolated.

What evidence there is suggests that the rhinoviruses isolated by organ culture are generally of the same serotype as those detected in tissue culture and their distribution among the various clinical diagnoses is similar (Higgins, 1966b). It is reasonable, therefore, to assume that the proportion of the total rhinovirus infections found in each of the clinical categories in these surveys is substantially correct although their relative importance compared to the other respiratory viruses has been underestimated. Similarly, the frequency with which certain clinical features were found in association with rhinovirus infections in these studies is probably true and unlikely to have been modified had a larger number of rhinoviruses been isolated by the use of additional methods.

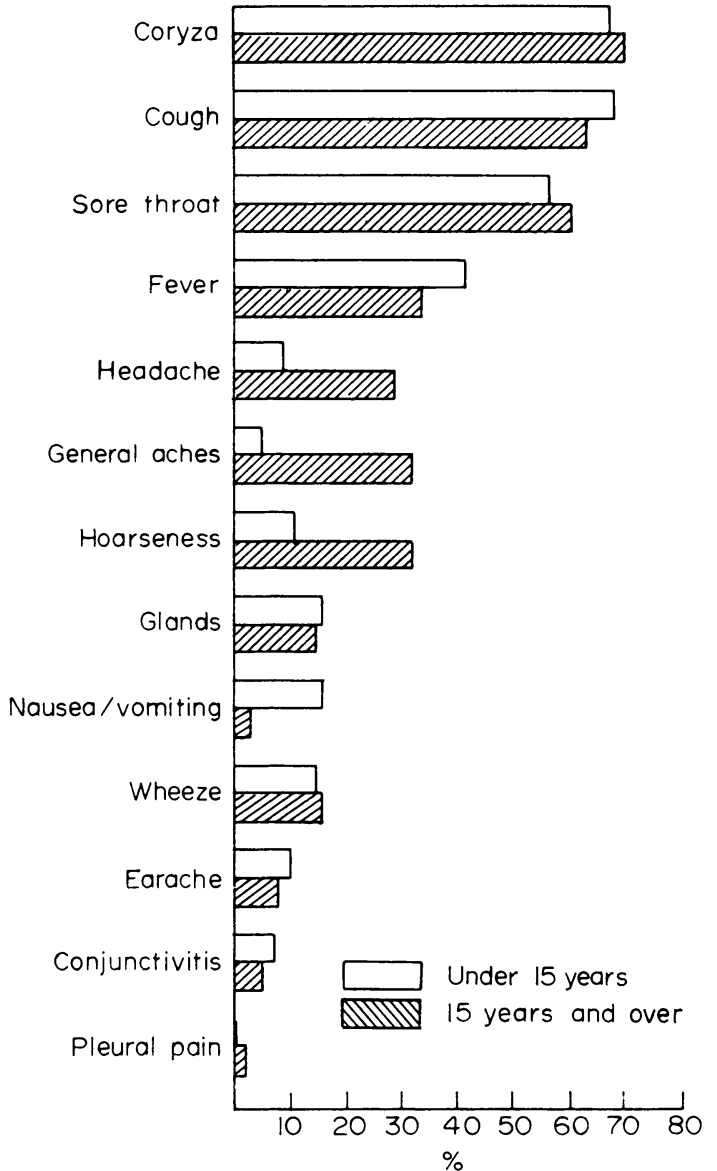

FIG. 4. Clinical features of sixty-six rhinovirus infections in children and 117 infections in adults. General practice survey.

Although these surveys have added little to our knowledge of the behaviour of the rhinoviruses they have succeeded in confirming some of the observations which have been reported since the commencement of these studies.

\section{References}

Hamre, D., Connelly, A.P. \& Procknow, J.J. (1966) Virological studies of acute respiratory disease in young adults. IV. Virus isolations during four years of surveillance. American Journal of Epidemiology, 83, 253.

Higgins, P.G. (1966a) The isolation of viruses from acute respiratory infections. Part IV. A comparative study of the use of human embryo kidney and human embryo diploid fibroblasts (W138). Monthly Bulletin of the Ministry of Health and the Public Health Laboratory Service, 25, 223.

Higgins, P.G. (1966b) The isolation of viruses from acute respiratory infections. Part V. The use of organ cultures of human embryonic nasal and tracheal ciliated epithelium. Monthly Bulletin of the Ministry of Health and the Public Health Laboratory Service, 25, 283. 
Higgins, P.G. (1967) The rhinoviruses. The Practitioner, 199, 633.

Higgins, P.G., Boston, D.G. \& Ellis, E.M. (1964) The isolation of viruses from acute respiratory infections. II. A study of the isolates made from cases occurring in a general practice in 1963. Monthly Bulletin of the Ministry of Health and the Public Health Laboratory Service, 23, 93.

Higgins, P.G. \& Ellis, E.M. (1973) Further observations on the use of organ cultures in the study of acute respiratory infections. Journal of Medical Microbiology, 6, 177.

Higgins, P.G., Ellis, E.M. \& Woolley, D.A. (1969) A comparative study of standard methods and organ culture for the isolation of respiratory viruses. Journal of Medical Microbiology, 2, 109.
Kendall, E.J., Bynoe, M.L. \& Tyrrell, D.A.J. (1962). Virus isolations from common colds occurring in a residential school. British Medical Journal, 2, 82.

Medical Research Council Working Party on Acute RESPIRATORY INFECTIONS (1965) A collaborative study of the aetiology of acute respiratory infections in Great Britain 1961-4. British Medical Journal, 2, 319.

TYRRELL, D.A.J. \& BYNOE, M.L. (1966) Cultivation of viruses from a high proportion of patients with colds. Lancet, $\mathbf{i}$, 76.

Tyrrell, D.A.J. \& Parsons, R. (1960) Some virus isolations from common colds. III. Cytopathic effects in tissue culture. Lancet, i, 239. 